\title{
Los linderos de la investigación en México: experiencias en territorios inseguros
}

\author{
The boundaries of research in Mexico: Experiences in dangerous zones
}

\author{
RubÉn DARÍo Ramírez SÁNCHEZ* \\ doi.org/10.29043/liminar.v20i2.911
}

Resumen: El presente trabajo tiene por objetivo mostrar algunas implicaciones epistemológicas y metodológicas que se presentan al realizar investigación en territorios inseguros en México. Se empleó un método de análisis antropológico y sociológico que ubica la violencia como objeto de conocimiento, con nuevas rutas de investigación, estrategias etnográficas y de análisis de la información. Estas rutas y estrategias se han incorporado a partir de experiencias investigativas que tuvieron lugar en diversos territorios inseguros del país. El trabajo permite concluir que los contextos inseguros han propiciado que el quehacer investigativo tome nuevas direcciones epistemológicas y metodológicas para abordar fenómenos derivados de la violencia criminal, comprendida conceptualmente en el marco de una nueva guerra librada entre los cárteles de la droga y contra el Estado.

Palabras clave: investigación, violencia, territorios inseguros, trabajo de campo.

Abstract: This paper presents various epistemological and methodological implications associated with research in dangerous regions in Mexico. Anthropological and sociological analysis methodologies were used to study violence as an object of knowledge, with new research routes and new ethnographic and information analysis strategies. These routes and strategies have been incorporated from research experiences in various dangerous territories in Mexico. This study concludes that dangerous contexts have led researchers to take new epistemological and methodological routes to address phenomena derived from criminal violence, conceptually understood within the framework of a new war between drug cartels and the state.

Key words: research, violence, dangerous territories, fieldwork.

Recibido: 26 de abril de 2021

Aprobación: 5 de julio de 2021

Publicación: 18 de febrero de 2022

\author{
* Unidad Académica de Estudios Regionales \\ de la Coordinación de Humanidades \\ de la Universidad Nacional Autónoma de México, México \\ rubendario105@hotmail.com \\ (iD) 0000-0002-8766-0233
}




\section{La violencia como campo de estudio}

$\mathrm{L}$ a violencia como objeto de estudio ha sido abordada desde múltiples ángulos sociológicos. Desde autores como Durkheim, Weber, Talcott Parsons y Lewis Coser, hasta Michel Foucault, entre otros, han destacado la importancia que tienen las condicionantes estructurales y morales para comprender las distintas manifestaciones de este fenómeno (Arteaga y Arzuaga, 2017). Dada la diversidad semántica que ha acompañado la reflexión en torno a la violencia, considero necesario hacer algunas precisiones conceptuales que permitan tener un panorama general. En primer lugar, coincido con Gilberto Giménez cuando sostiene que "no existe la violencia en abstracto ni como concepto inequívoco, porque se trata de un fenómeno complejo, heterogéneo y pluridimensional difícil de atrapar en un concepto" (Giménez, 2017, p. 10). Es por ello que al estudiarla se debe considerar su sentido plural, ahondar en las causas, autores, víctimas, lugares y circunstancias que la potencian o detonan, así como establecer escalas, clasificaciones o tipologías, conocer las conexiones estructurales que permitan teorizarla, y analizarla junto a otros fenómenos sociales.

En segundo lugar, derivado de la diversificación de los fenómenos criminales, el concepto de violencia es polisémico, aunque se acentúan dos posiciones de debate. Por un lado, está la perspectiva que la considera como una acción que va más allá de la aplicación de fuerza para hacer daño y "constituye una desviación (legal, moral o social)" (Jackman, 2002, p. 338). Esta visión deriva de una idea centralista del Estado, que lo considera el detentador hegemónico del uso legítimo de la violencia. Otra posición, con la cual coincido, es la que pondera la dimensión social y cultural de estos fenómenos, además de su "fisicalidad", debido a que son estas dimensiones las que le confieren poder y sentido (Scheper-Hughes y Bourgois, en Giménez, 2017, p. 15). Los sistemas de desigualdad social fungen como generadores de violencia privada, estatal y estructural, tal como sustenta Schinkel (2013); o de violencia subjetiva, simbólica y sistémica, como sostiene Žižek (2009). En consonancia con esta idea, Johan Galtung (2004) explica esta clasificación y sus conexiones a través del triángulo de la violencia. Para él, la violencia directa se encuentra en el vértice superior del triángulo. Esta se concreta en comportamientos y responde a actos concretos de violencia verbal, física o psicológica. En los ángulos de la base del triángulo están las violencias estructurales y las violencias culturales. Las primeras se refieren al conjunto de estructuras que no permiten la satisfacción de las necesidades y se concretan en la negación de ellas. Las segundas son las que crean un marco legitimador de la violencia y se concretan en actitudes.

Entender la violencia como un concepto polisémico, heterogéneo y multidimensional permite analizar de manera integral los conflictos, actores y procesos sociales que generan los cárteles de la droga. Un enfoque sistémico permite comprender dichos conflictos como nuevas guerras, que se caracterizan por ser "dispersas, descentralizadas, y de bajo nivel tanto de intensidad como de involucramientos militar, pero de mayor duración (que puede prolongarse durante años)” (Giménez, 2017, p. 22). Estamos ante una guerra de cárteles - configurados como empresas criminales jerárquicas con capacidad autónoma de acción, coerción e influencia sobre otros actores y que cuentan con una organización jerárquica que les permite desarrollar una actividad policriminal - que lidian entre sí y contra el Estado. Debido a que su clasificación resulta heterogénea, la violencia deberá pensarse en términos relacionales y analizarse en un contexto espacio-temporal, es decir en términos geohistóricos, y en el marco de un conflicto y de la historia de ese conflicto. De acuerdo con Rosemberg, "la violencia tiene diferentes rostros y el más 
evidente, el más explicado y observado es el de la guerra, pero la violencia no se agota en la guerra”, debido a que la violencia es social y doméstica, por lo cual "está en relación-interacción con varios ejes de su estructura-orgánica todos ellos entrelazados en una trama compleja” (2019, pp. 161-167).

En los últimos tres lustros, la disputa territorial entre los cárteles de la droga en México ha ocasionado que muchas regiones enfrenten condiciones de guerra, tal como sucede en el corredor que involucra a los estados de Durango, Sinaloa y Chihuahua, o el de Occidente que incluye los estados de Guanajuato, Jalisco, Estado de México, Guerrero y Michoacán. Otras ciudades como Tijuana en Baja California, Ciudad Juárez en Chihuahua, Minatitlán en Veracruz y Colima, la capital de Colima, viven álgidas escaladas de violencia regional. En Michoacán, sus regiones han enfrentado la expansión de la violencia desatada por la guerra que emprendió el gobierno federal de Felipe Calderón Hinojosa contra los cárteles de la droga en 2006. ${ }^{1}$ El resultado de esta álgida disputa territorial fue la fragmentación y expansión de los cárteles (Astorga, 2009) y el desvanecimiento de las fronteras entre quienes detentan legítimamente la autoridad y quienes desde la ilegalidad imponen su hegemonía (Maldonado, 2013). Al constituirse en una zona de guerra, la entidad atrajo la atención de centenares de reporteros, de medios nacionales y extranjeros, interesados en el conflicto. También, atrajo la atención del medio académico, principalmente de especialistas en ciencias sociales, con el objetivo de explicar tanto la emergencia, estructura y diversificación del narcotráfico, como la actuación del Estado, la configuración y el predominio de las economías criminales o sumergidas (Pontón, 2013). El estudio de la violencia se convirtió en un campo de análisis sociopolítico atractivo para la academia. De esa manera fue como surgieron nuevas líneas y proyectos de investigación, nuevos programas de estudio y observatorios. El resultado fue un número importante de informes y publicaciones, a grado tal que se considera que arribamos a una "sobre diagnosticación de la violencia" (Zavaleta, 2017, p. 45).

Los trabajos dirigidos a explicar este fenómeno en Michoacán pueden agruparse en dos etapas de tratamiento. En una primera etapa, que va del año 2006 al 2013, las investigaciones se enfocaron en estudiar fenómenos como el tráfico de drogas y su inserción en la economía regional (Maldonado, 2010), la declaración de guerra del gobierno federal, el proceso de atomización de los cárteles de la droga y sus efectos en el incremento de homicidios dolosos o crímenes de segundo Estado (Segato 2013), así como las distintas caras o fisonomías que el Estado presentaba frente a los desafíos de este Estado paralelo (Briscoe, 2008). ${ }^{2}$ Esto se debió a que la guerra emprendida por el gobierno federal provocó una permanente fragmentación y expansión de los cárteles de la droga, que ocasionó que muchas regiones del estado se convirtieran en escenarios de violencia crónica. ${ }^{3}$ Los "altos índices de violencia que se han mantenido durante varios años", es decir su consistencia en espacio, tiempo e intensidad, le dan a la violencia un

\footnotetext{
${ }^{1}$ En las seis regiones del estado de Michoacán se viven procesos diferenciados de violencia, aunque el punto más álgido es Tierra Caliente, área integrada por los municipios de Apatzingán, Zitácuaro, Nueva Italia, Buena Vista, Parácuaro, la Huacana, Tepacaltepec y Aguililla debido a la disputa territorial que tienen Cárteles Unidos y el Cártel Jalisco Nueva Generación. Otras ciudades, como Uruapan y Zamora, presentan altos índices de homicidios violentos y son consideradas de las más violentas en el país.

${ }^{2}$ Variadas discusiones se han generado en torno a la construcción del Estado frente a hegemonía regional del narcotráfico. Destacan las categorías de Estado débil, Estado fracasado (Tedesco, 2012) y Estado fallido (Rotberg, Clapham y Herbst, 2007), las cuales dan cuenta de las distintas formas o caras que este puede asumir frente a la expansión del narcotráfico.

${ }^{3}$ Del año 2006 al 2019, salvo en el periodo entre 2009 y 2012, la tasa anual de homicidios en Michoacán superó los veinte homicidios por cada cien mil habitantes, por encima de la media nacional. Tras la intervención del gobierno federal, de 2016 a 2019, los índices ascendieron a 27.5, 26.2, 26.0 y 24.2, respectivamente (Estrada, Hinojosa y Badillo, 2019).
} 
sentido sistémico (Pearce, 2019, pp. 11-12). Esta violencia, crónica y sistémica se reproduce a partir de micro y macroprocesos insertos en las estructuras sociales, que fragmentan y afectan la capacidad social de reactuación. Esto le confiere, además, un carácter transversal, multidimensional y multifactorial, donde la impunidad funge como el principal mecanismo para su reproducción (Zavaleta, 2017; Ramírez, 2019a).

En una segunda etapa, que comprende de 2014 a 2018, los trabajos se enfocaron en explicar la emergencia de las autodefensas y otros grupos movilizados en los márgenes del Estado. La radiografía analítica mostraba cómo algunos de aquellos grupos cumplieron una función de resguardo de sus poblaciones, en tanto que otros fungieron como brazos activos de los cárteles de la droga (Pansters, 2019; Le Cour, 2019). En tal sentido, las reflexiones estuvieron dirigidas a comprender cómo la construcción de esta territorialidad conflictiva engendró un proceso gradual de normalización y banalización de la violencia, y la convirtió en un espacio de socialización y reconfiguración de las relaciones sociales (Maldonado, 2018 y 2019). Asimismo, analizaban cómo este proceso dio paso al establecimiento de una gobernanza criminal, la cual se implantó a partir de que los grupos criminales lograron "establecer instituciones para controlar las relaciones de las poblaciones establecidas en su área de control territorial” (Azula, 2017, p. 10).

Aunque en el pasado reciente muchos proyectos de investigación estuvieron dirigidos a estudiar la presencia de los cárteles de la droga y las excentricidades de sus principales dirigentes, el crecimiento exponencial de la violencia y sus diversas articulaciones con el proceso de construcción social dieron paso a la emergencia de múltiples objetos de estudio en el campo de las ciencias sociales en México. ${ }^{4}$ Los efectos de la violencia empezaron a ejercer una influencia directa en los procesos de desarrollo regional y de transición política, lo cual abrió rutas de análisis para explicar la nueva correlación de fuerzas y la formación de lo que aquí será denominado territorios inseguros. En mi experiencia investigativa, el arribo a este campo se debió a que durante el desarrollo del proyecto "Transición política partidaria y cultura política en la región Ciénega de Chapala” las manifestaciones de la violencia fueron cada vez más persistentes y definitorias en la vida regional, por lo que fue incluida como una vertiente de análisis en el proyecto. Al mismo tiempo, se ampliaron los parámetros investigativos hacia otras regiones de Michoacán, particularmente la de Tierra Caliente. ${ }^{5}$ Por ese motivo fue necesario replantear la investigación hacia "La construcción del Estado en territorios inseguros en Michoacán y la configuración de una democracia violenta". ${ }^{3}$ Este giro en el objeto de estudio se debió a que la consolidación territorial del narcotráfico

\footnotetext{
${ }^{4} \mathrm{Al}$ respecto, Veena Das (1997, p. 88) considera que los estudios de las ciencias sociales deben considerar "el dolor de los otros, las emociones de este tipo de relaciones sociales y del sentido de la acción humana". Ello implica reconocer el estado emocional de los actores, el contenido cultural de las emociones, su lugar en la cultura particular para recobrar una dimensión ignorada de la acción social. Por lo anterior, plantea la necesidad de apelar a la "experiencia de violencia", la cual nos permitirá desentrañar los significados, motivaciones, emociones prácticas corporales y discursivas de los sujetos en los actos de violencia.

${ }^{5}$ Estos cambios de dirección investigativa nos obligaron también a ahondar en los diferentes estudios que la literatura antropológica y sociológica había producido en las últimas dos décadas. En el plano sociológico encontramos trabajos como los de Guillermo Trejo y Sandra Ley (2015), entre otros, que ofrecen una importante ruta de análisis cualitativo. Dichos trabajos explican la redistribución del poder y el comportamiento sociopolítico regional a partir de la agudización de la violencia. En el plano antropológico encontramos aportes como el de Wil G. Pansters (2019), quien propone un estudio descentrado del Estado. Sostiene como tesis central que este se construye mediante la interrelación en la vida cotidiana de múltiples actores legales e ilegales.

${ }^{6}$ Durante el desarrollo del proyecto "Transición democrática y cultura política en la región Ciénega de Chapala" se encontraron muchos vasos comunicantes con la violencia ligada al narcotráfico como actor decisorio en la geografía del poder local, por lo cual se tuvo que reorientar el trabajo hacia la "Inseguridad y construcción del Estado en Michoacán".
} 
convirtió las regiones en zonas difusas o grises. ${ }^{7}$ Estas zonas son lugares donde coexisten bandas locales independientes o ligadas a cárteles nacionales de la droga, grupos de autodefensas o autoridades locales y federales. En este cambio influyó también mi condición de habitante de la Ciénega de Chapala, donde la autoridad "cambió de mano" o se subordinó a los cárteles de la droga, y donde tuvo lugar un amplio proceso de resiliencia social en estas poblaciones para ajustarse y aprender a vivir en riesgo. ${ }^{8}$

La comprensión de los distintos ángulos e interconexiones que presenta la violencia derivada del narcotráfico en la construcción regional requirió del diseño de una ruta metodológica, la cual se articula a partir de los siguientes cuatro ejes de análisis. El primero reside en considerar el narcotráfico como un fenómeno transnacional. Este fenómeno es consecuencia de la apertura económica y la desregulación neoliberal, principalmente asociada a la firma del Tratado de Libre Comercio de América del Norte (TLCAN) y a las transformaciones de los canales de introducción de la cocaína en Estados Unidos y el tráfico de armas (Bataillon, 2015). El segundo consiste en identificar analíticamente la importancia de los anclajes fronterizos, que por su porosidad facilitan los circuitos de tránsito y el despliegue interestatal de los cárteles de la droga (Astorga, 2009; Maldonado, 2014). El tercero implica considerar los efectos del proceso de transición democrática y la fragmentación del sistema político en la construcción de una nueva territorialidad criminal. En este contexto el Estado tiene una presencia testimonial, mantiene connivencia con los cárteles o está ausente. El punto más álgido de este fenómeno se produjo en el año 2011, cuando alrededor de 80 de los 113 municipios del estado y algunas instituciones del gobierno estatal fueron infiltradas total o parcialmente por estos grupos. ${ }^{9}$ En el cuarto eje se considera analizar la emergencia y expansión de los grupos de autodefensas en 2013, así como su institucionalización en guardias rurales en 2014 y los efectos del establecimiento de una estructura paralegal del gobierno federal. El resultado fue la fragmentación de los cárteles locales, que ha conducido a la actual agudización de la disputa territorial entre los Viagras, remanentes de los Caballeros Templarios, la Nueva Familia Michoacán y una decena de grupos locales integrados en la agrupación Cárteles Unidos para impedir que el Cártel Jalisco Nueva Generación ingrese al estado.

\footnotetext{
${ }^{7}$ Son espacios intermedios donde los límites entre lo legal y lo ilegal se entrecruzan, las fronteras se difuminan y los contendientes se multiplican. Los contornos mal definidos separan y unen a los bandos involucrados; la piedad y la brutalidad pueden coexistir en el mismo individuo o grupo y en el mismo momento contra toda lógica (Auyero, 2007).

${ }^{8}$ En estas condiciones de violencia y ante la deficiencia o ausencia de las instituciones del Estado — aunado a que el narcotráfico se convirtió en una actividad ocupacional con distintas tareas y grados de especialización criminal- la población ha aprendido a resolver sus necesidades al margen de dichas instituciones. A menudo no se puede hacer de otra manera, fundamentalmente porque hay regiones - como sucede en municipios de Tierra Caliente, Michoacán, como Aguililla, Apatzingán, Felipe Carrillo Puerto (La Ruana), Tepalcatepec y Los Reyes-, donde grupos legales e ilegales han establecido horarios o suspendido el libre tránsito. En algunas regiones no solo se ajustan a la presencia y acción de "la maña", sino que además se les percibe como una especie de autoridad local. Recurren a ella, por ejemplo, para el otorgamiento de créditos, el financiamiento de festividades locales, reportar sospechosos de delitos comunes para que sean castigados, cuando se producen agravios de terceros, para preguntar por desaparecidos, y hasta para hacer solicitudes de mujeres que acuden a ella para aleccionar a sus esposos violentos o infieles (Bataillon, 2015).

${ }^{9}$ En coincidencia con González (2014), como sucedió en Colombia, en algunas regiones de México, entre ellas Michoacán, estamos frente a una "reconfiguración cooptada del Estado", la cual se da a partir de la confluencia de actores legales e ilegales con resultados disímiles para unos y otros. Nos encontramos ante una construcción regional del Estado que combina entornos descentralizados con débil institucionalidad, donde las instituciones estales y municipales son infiltradas y mantienen algún tipo de relación de connivencia con los cárteles de la droga.
} 


\section{Investigación en zonas difusas}

$\mathrm{Al}$ convertirse la violencia en un campo de conocimiento, ${ }^{10}$ su estudio se ha tenido que desarrollar en condiciones críticas asociadas a la incertidumbre y al miedo transversal que esparce. Esto ha propiciado que algunas investigaciones se interrumpan, cambien de rumbo o se cancelen. La nueva territorialidad violenta afecta directamente la construcción del conocimiento, no solo en la manera de leer los entramados de esta realidad, sino en la forma en que la persona que investiga enfrenta los riesgos.

Trabajar en estas circunstancias representa un proceso de aprendizaje que obliga a quien investiga a entender la violencia no solo como una expresión más de la lucha por el poder, sino como un "campo de saber y poder" (Jiménez-Ocampo, 2008). Exige también explorar nuevos métodos de lectura de esta red de relaciones locales reguladas por diversos regímenes de violencia. Sin embargo, la puesta en marcha de los proyectos de investigación dirigidos a deshilvanar los procesos, actores y víctimas de la violencia ha propiciado dos cuestionamientos. El primero se refiere a la manera en que algunas disciplinas de las ciencias sociales, como la sociología, la antropología o la ciencia política, entre otras, abordan la violencia. ${ }^{11} \mathrm{El}$ segundo cuestionamiento se refiere a los límites que el quehacer investigativo presenta al abordar realidades exacerbadas por las tensiones vivenciales, es decir, de qué forma "nuestros temores a ella tienen efectos reales en las maneras de interpretar la realidad" (Maldonado, 2013, p. 64). ${ }^{12}$

Frente a estos dilemas proponemos una comprensión integral de la violencia más allá de las barreras disciplinares y mediante metodologías flexibles que permitan comprenderla-aprehenderla no solo como el acto de infringir fuerza para dañar al otro, sino como una trama compleja cuya relación social se caracteriza por "la negación del otro" (Martínez, 2016, p. 16); que no "agota lo social ni lo destruye", sino que "produce saberes y sujetos, tiene una nueva 'sociabilidad", donde "las prácticas de victimización dependen de posicionamientos móviles en la correlación de fuerzas, que el monopolio de la violencia legítima es una construcción que produce sus contrapartes ilegales" (Agudo, 2011, en Zavaleta, 2017, p. 51). Entendida de esta manera, la violencia se distingue como un factor influyente en la construcción regional.

Con el objetivo de mitigar los riegos, se consideró necesario identificar los códigos y las lógicas de la violencia que conducen la vida en estas poblaciones. También fue necesario explorar las actuales rutas de análisis para leer el nuevo paisaje rural michoacano. Es una región habitada por poblaciones mayoritariamente empobrecidas, que se han construido históricamente sobre los basamentos de la interme-

\footnotetext{
${ }^{10}$ La inseguridad y la violencia derivadas del narcotráfico han impactado sobre muchos de los procesos sociales y económicos de los que emanaban los objetos de estudio de la sociología y la antropología social. Ello nos sitúa frente a nuevas condiciones materiales donde se construye la vida social. Estas dinámicas han conducido a quienes investigan a incursionar en nuevos campos o a reconstruir los objetos de estudio. Esto exige poner atención en las nuevas relaciones de los sujetos y desarrollar una mayor acuciosidad para aprehender las nuevas dinámicas y rumbos que esta construcción social toma.

${ }^{11}$ Se ha planteado que el problema central de las diversas disciplinas que estudian la violencia es la falta de definición precisa que articule o consensue la multiplicidad de formas y la diversidad de expresiones que manifiesta. Se argumenta que al ser estudiada desde diversos campos de conocimiento se "dificulta no sólo su estudio en general, sino la aceptación de una definición clara y unívoca”. Sin embargo, es necesario precisar que esta diversificación semántica ha contribuido a la exploración de su complejidad y causalidades, su morfología y su dinámica (Martínez Pacheco, 2016, p. 7).

${ }^{12}$ Un cuestionamiento adicional, con el cual coincido, es el que hace Nordsfrom (1997, p. 17), quien considera que "escribir sobre las experiencias de violencia de personas de carne y hueso, es más difícil que hacerlo sobre la violencia per se. La violencia no puede separarse de la acción, la violencia política es ejercida a alguien por alguien".
} 
diación caciquil (Castro, 2017). Son pobladores que intercalan sus vidas entre México y Estado Unidos y que, debido a la presencia del narcotráfico en su cotidianidad, han entrado en un amplio proceso de reconstrucción y resignificación.

$\mathrm{Al}$ abordar este campo de estudio y entrar en contacto con zonas inseguras - tales como la región de la Ciénega de Chapala y la Tierra Caliente, en Michoacán - surgió la necesidad de modificar tanto el modo de acercarse a los pobladores, como la forma de analizar la información y construir las narrativas. En muchas ocasiones, por ejemplo, fue necesario omitir nombres de lugares y personas. Esto se debe, primordialmente, a que la continuidad de la guerra calderonista durante el gobierno de Enrique Peña Nieto (2012-2018) expandió las restricciones del libre tránsito. Proliferaron los retenes de vigilancia establecidos por grupos esbozados de autodefensas o cárteles de la droga. Esta situación incentivó el desplazamiento de personas hacia regiones más seguras, o a Estados Unidos, y se restringió el ingreso de visitantes (Castro, 2017). ${ }^{13}$ La dificultad que representa transitar y habitar en estas poblaciones exigió buscar alternativas de aproximación en esta nueva geografía del poder.

En la búsqueda de caminos para explicar las dinámicas dentro de territorios inseguros nos enfrentamos a desafíos epistemológicos, ético-políticos y etnográficos (de estos últimos me referiré en un apartado posterior). En el terreno epistemológico enfrentamos complicaciones a la hora de objetivar y teorizar; fundamentalmente, cuando intentamos guardar distancia de la realidad, de los asuntos y circunstancias del lugareño, y nos colocamos en esa enorme "zona de no ser". Esto se debe a que nuestra presencia en los espacios nos convierte en un actor más que enfrenta diversas presiones, las cuales nos obligan incluso a movernos en las sombras, a repensar, reorientar o abandonar nuestra investigación.

Inicialmente, la investigación que realicé estaba dirigida al estudio de la transición democrática y la construcción del Estado en la región Ciénega de Chapala, Michoacán. Sin embargo, la dinámica comercial y la ubicación fronteriza de esta región con el estado de Jalisco la convirtieron en una zona estratégica para el comercio de mercancías ilícitas y el tráfico de drogas sintéticas. Otras incursiones en la región de Tierra Caliente, frontera con Guerrero y el Estado de México, me permitieron observar la disputa territorial entre los cárteles de la droga e incorporar nuevos elementos de análisis a mi objeto de estudio inicial. En ambas incursiones observé cómo en los últimos años los grupos ilegales ligados al narcotráfico se convirtieron en actores importantes en la construcción del poder local en estas regiones. Con ello, tanto la transición política como la construcción del Estado tomaron nuevos rumbos, dado que son procesos articulados. Así fue como el trabajo de investigación se redirigió a reflexionar sobre la configuración de un Estado dentro de otro Estado o "Estado paralelo" (Segato, 2013, p. 29). Para ello fue necesario ubicar y comprender las distintas construcciones estatales a partir de estos poderes ilegales (Castro, 2017, p. 67), dentro de las cuales el Estado tiene también una actuación ilegal o "en las sombras" (Gledhill, 2008). Esta actuación ilegal sucede incluso contra la propia sociedad civil. Las referencias ilícitas más significativas las identificamos en la represión que ejercieron las fuerzas policiales federales en contra de los grupos de autodefensa, en Apatzingán y Tanhuato, y contra comuneros de la comunidad de Arantepecua, en Nahuatzen (Castellanos, 2015; Ramírez, 2020).

\footnotetext{
${ }^{13}$ En México, la Comisión Mexicana de Defensa y Promoción de los Derechos Humanos (CMDPDH) calculó que entre 2006 y 2018 hubo 338405 personas desplazadas. En Michoacán, entre estos mismos años se desplazaron 11590 por la violencia, y más $80 \%$ del desplazamiento se produjo en las zonas rurales. En 2019 Michoacán ocupó el tercer lugar en cuanto a desplazamientos forzados por la inseguridad, detrás de Guerrero y Oaxaca (Gutiérrez, 2020).
} 
Explicar esta compleja construcción social y sus dinámicas conflictivas condujo la investigación a analizar los espacios abiertos por el proceso transicional. Este proceso de transición fue aprovechado por los cárteles de la droga para posicionarse tanto en la geografía político partidaria como en la construcción regional del Estado, donde participan actores legales e ilegales. Esta tarea ha obligado a descentrar el análisis del Estado (Bonilla en Rotberg, Clapham y Herbst, 2007) ${ }^{14}$ con el fin de explicar teórica y empíricamente el comportamiento de este en un contexto cruzado transversalmente por la violencia ilegal en una pluralidad de regímenes. En este sentido, el concepto de Estado no se aborda como "cosa", sino como una construcción heterogénea donde participan actores legales e ilegales.

Explicar este fenómeno ha tenido implicaciones importantes en el proceso de observación, interacción y análisis. El contacto directo con esta empiricidad violenta propicia lo que Castro denomina una "economía emocional" (2017, p. 68), la cual se da a partir del cruzamiento de todo tipo de miedos, impotencias, frustraciones, enojo e indiferencia. Esto se debe a que como sujeto investigador no es posible escindirse de los efectos emocionales que propicia la escena investigativa en la que participamos. Es necesario, entonces, revalorar la estrategia metodológica y los instrumentos de recolección de información regularmente utilizados en contextos no violentos. Puede hacerse necesario prescindir del uso de algunas herramientas tecnológicas, tales como las grabaciones o captura de imágenes y las anotaciones públicas, lo que conduce a hacer de la memoria un recurso indispensable para el registro de información.

En lo respectivo a las motivaciones éticas y políticas que acompañan el hacer investigación en estos territorios, corresponde bosquejar por lo menos tres planteamientos. El primero radica en advertir la dificultad que enfrentamos para reflexionar sobre la posición que asumimos como actores en el drama de la violencia (Rosemberg, 2019), y la responsabilidad que tenemos de plantear nuestros intereses a los involucrados en el proceso investigativo, bajo la premisa de no irrumpir ni confrontar los viejos y nuevos códigos de convivencia establecidos en la población de estudio. Ello implica mantener una distancia prudente, que emane de una lectura estratégica del contexto y que no confine la confianza con los involucrados. Una distancia que nos permita identificar las circunstancias políticas y la correlación de fuerzas a nivel local frente a las instituciones del Estado y al interior de sus propias comunidades (Castro, 2017). También, será útil advertir los entrecruces de interés, las relaciones o los juegos de poder que determinan las narrativas y el comportamiento de los actores que intervienen en la construcción local del Estado. En esta tarea resultará determinante reconocer los sesgos que estos contextos riesgosos generan y la forma en que podemos lidiar con ellos. La carga emocional que produce el tratamiento de este fenómeno es un incentivo latente que puede limitar o potenciar el proceso reflexivo. De ahí la necesidad de estar alertas a la influencia que nuestras empatías - determinadas por posiciones ideológicas o de clase- puedan ejercer en la construcción narrativa.

El segundo planteamiento radica en advertir los efectos de nuestra posición de poder. La condición de investigador ${ }^{15}$ nos otorga una amplia capacidad de decisión sobre la selección de la información, que

\footnotetext{
${ }^{14}$ Un análisis destacado de esta posición lo presenta Daniela Bonilla (2007), quien analiza las distintas discusiones sobre el Estado fallido. Destacan también las aportaciones de Robert I. Rotberg (en Rotberg, Clapham y Herbst, 2007), defensor de la "escuela conservadora de los estados fallidos", quien reflexiona sobre los distintos niveles de debilidad estatal.

${ }^{15}$ La figura del sujeto investigador ha entrado en una especie de impasse debido a la idea o referencia que socialmente se ha construido en torno al quehacer policial, por lo cual resulta determinante cambiarlo o camuflarlo con el de "profesor", cuya figura todavía es confiable.
} 
en ocasiones pone en riesgo a los involucrados en el proceso de investigación. En tal sentido resulta indispensable, como menciona Massey, "diagramar de manera consciente y autocrítica la propia geometría de poder del etnógrafo, como criterio de ingreso para poder eventualmente detectar, trabajar y reconvertir los sesgos en material para el análisis etnológico y/o reflexivo", para adecuar "los intereses y prioridades heurísticas, como a los principios éticos tanto a nivel político como metodológicos" (en Castro, 2017, p. 83). Un ejercicio de esta envergadura posibilitará la construcción de relaciones de reciprocidad y condiciones de seguridad para quienes participen en el proceso de investigación, que nos permita mostrar cómo la violencia nos modifica, cómo se transforman las relaciones dentro de la comunidad o el grupo con el que trabajamos en el campo, y al mismo tiempo cómo "el trabajo de campo requiere de la experiencia vivida de la violencia y las formas de conocer y reflexionar sobre la violencia" (Rosemberg, 2019, p. 168).

El tercero plantea la necesidad de un pensar estratégico encaminado a eludir los riesgos en estos entornos de violencias entrecruzadas. Este ha de ser un pensamiento que nos permita advertir las amenazas, controlar los efectos de la violencia y eludir o lidiar con la "paranoia y ostracismo etnográfico" o el "shock existencial, que desestabiliza la dialéctica entre empatía y distanciamiento" (Nordstrom y Robben, 1995, en Ferrándiz y Feixa, 2004, p. 165). Ello implica mantener estabilidad emocional para generar estrategias de protección y autorregulación que nos permitan realizar nuestro quehacer investigativo. Una vía para lidiar con ello es acudir a la experiencia de otros colegas que trabajen en territorios inseguros. También, es necesario diseñar protocolos derivados de nuestras prácticas que emanen de los procesos de investigación en estos contextos (Castro, 2017).

\section{Información e imagen de la violencia}

Otro desafío que enfrentamos cuando se busca comprender contextos de este tipo es el tratamiento que le damos al torrente de información que vierten los medios de comunicación y las redes sociales sobre la violencia. Las noticias recurrentes sobre "ajustes de cuentas" mediante ejecuciones grupales o individuales conforman una extensa iconografía de cuerpos rotos y de fosas clandestinas. En ellas se muestra la vulnerabilidad del otro, "el enemigo inerte" abatido y despojado de su condición humana (Cavarero, 2009, p. 43). ${ }^{16}$ La exposición de esta realidad muestra escenas e historias de horror que alimentan el terror cotidiano y dan forma a lo que Bourgois (2010) denomina la pornografía de la violencia. También lo describe como "el uso promiscuo y descontextualizado que tiene como consecuencia la banalización de los hechos y del sufrimiento social que les acompaña" (Ferrándiz, 2008, p. 110). Aunada a esta atroz diseminación de información, cuya exposición espectacular y dramática nutre nuestros temores, enfrentamos también lo que Maldonado (2013, p. 66) ha denominado la "estatización del discurso de la violencia”. ${ }^{17}$ Con ello se refiere a la forma en la que el Estado se extrae de la violencia. Lo lleva a cabo

\footnotetext{
${ }^{16}$ La violencia por la disputa territorial en la Ciénega de Chapala propicia una letalidad difícil de cuantificar por el hermetismo que guarda la autoridad local. Es difundida en las redes sociales, en tanto que regularmente está ausente en los medios de comunicación impresos, radiofónicos o televisivos.

${ }^{17}$ La estatización hace referencia "tanto a los aparatos formales del Estado como a aquellos vasos capilares que estructuran y dan sentido al poder social. La estatización es una forma de gubernamentalidad en el sentido foucaultiano, de producción de orden, de gobernar en razón de dirigir" (Maldonado, 2014, p. 66).
} 
mediante el control sobre la producción del discurso referente a la violencia y sobre la diseminación de la información oficial, de las cuales se valen los periodistas, observatorios ciudadanos y organizaciones no gubernamentales para remitir sus notas o informes.

Sin embargo, esto no demerita el valor de las fuentes de información estatal. Aunque su origen puede representar un sesgo, es nuestra responsabilidad corroborar y triangular críticamente datos obtenidos "en un medio social contaminado por la violencia" (Rosemberg, 2019, p. 166), para construir argumentos de explicación sólidos sobre tales realidades. En esta tarea también se debe advertir la repercusión que tiene sobre nosotros el manejo de este afluente de información. Hacer una lectura de hechos violentos nos ubica en una delgada línea emocional que puede sesgar nuestras narrativas. Esto se debe a que en el ejercicio reflexivo lidiamos con nuestra ansiedad etnográfica por realizar una lectura crítica del espectáculo y de la trivialización mediática de la violencia. Para ello se requiere de un entrenamiento emocional paulatino que nos permita tomar decisiones respecto a la pertinencia del uso de técnicas, el contacto y la elección de informantes.

En los últimos tres lustros, la disputa territorial que libran los cárteles de la droga ha multiplicado los "crímenes de segundo Estado" (Reguillo, 2012, pp. 34-35) y ha propiciado que la letalidad criminal aumente. ${ }^{18}$ También, ha ocasionado que la violencia se consolide como un "dispositivo de modelaje, aprendizaje y disciplinamiento de los sujetos" (Reguillo, 2012, p. 37), mediante la generación de estructuras de dominación y la diseminación de valores y normas (Cisneros, 2015). En esta construcción de conductas violentas, el uso ilegal de la fuerza se expresa como "violencia directa" y se asienta en la "violencia estructural", que es producto de "las desigualdades y condiciones de vida" (Rodríguez, 2012, p. 44). ${ }^{19}$ Afianzadas en la impunidad orgánica que es validada, legitimada y normalizada en las reglas de convivencia, se toleran los feminicidios, las ejecuciones extrajudiciales, las fosas clandestinas, la corrupción de las instituciones de seguridad y la vulnerabilidad de las víctimas (Cisneros, 2015).

Este entrecruzamiento de violencias, de acuerdo con Reguillo (2012, p. 37), construye una atmósfera de miedo y horror. Analíticamente, se explica a partir de la medición objetiva de las víctimas y los efectos de la violencia que hacen las autoridades oficiales; también se explica a través de la "violencia subjetivamente percibida" o la sensación expansiva que la gente percibe de la violencia "y su capacidad de producir lo social". Estas formas de percibir las violencias construyen atmósferas de miedo y horror que contribuyen a la erosión de la distinción entre "el vulnerable y el matable" (Cavarero, 2009, p. 60). Su efecto paraliza, incomunica y reduce las relaciones comunitarias que generan entornos de seguridad en la población (Ramírez, 2019b). Es así como en este hilado de procesos se configuran escenarios violentos y dinámicos, lo que complejiza su aprehensión y explicación. Esto ha ocasionado que muchas investigaciones se hayan dirigido a discernir los significados del terror sin dar suficiente preeminencia al contexto histórico, el cual da sentido y significado a estas realidades.

\footnotetext{
${ }^{18}$ En la última década, en Michoacán se produjo un crecimiento exponencial de homicidios dolosos de 161\%, ya que en 2009 se registraron 936 crímenes y en 2019 se contabilizaron 2 448. Estas cifras son el motivo por el cual Michoacán se ha mantenido entre los cinco estados más violentos del país (En la última década..., 2020).

${ }^{19}$ Michoacán se ubica como el tercer estado con más violencia familiar, al registrar en promedio 5000 denuncias al año. En lo que respecta a la violencia contra las mujeres, experimentó un crecimiento de $46.5 \%$ en todos los rubros. Las cifras pasaron de 902 mujeres denunciantes en 2019 a 1322 hasta agosto de 2020. Se registraron 14 feminicidios y 169 homicidios dolosos contra mujeres de enero a septiembre (quinto lugar nacional), además de3 018 víctimas de lesiones dolosas (tercer lugar nacional), 339 presuntos delitos de violación y 4393 llamadas por incidentes de violencia (SESNSP, 2020).
} 
Esta reconfiguración de la vida regional bajo la lógica de la violencia también nos obliga a lidiar con los códigos de silencio que se establecen como mecanismos de control. Estos códigos ponen a prueba la capacidad heurística de quien investiga para leer el contexto y moverse estratégicamente en poblaciones donde no es posible hablar de quien genera la violencia y sus víctimas.

Entre los años 2013 y 2016 recorrí las regiones michoacanas de la Ciénega de Chapala para explicar el proceso transicional y la de Tierra Caliente para conocer el fenómeno de las autodefensas. En 2018 recorrí la región Chontalpa de Tabasco, frontera con Veracruz, para observar la emergencia de las autodefensas tabasqueñas y el fenómeno del tráfico de gasolina, conocido como huachicol. ${ }^{20}$ En los tres casos realicé visitas esporádicas de ida y vuelta acompañado de colegas, amigos o habitantes conocidos. Con ellos transité por estas zonas, en ocasiones sin preguntar. Solamente observábamos los procesos de construcción social ilegal en territorios bajo la hegemonía de la delincuencia organizada y desorganizada, que se disputaba el mercado ilegal y la plusvalía derivada de esta actividad económica subterránea.

La observación y la observación participante son una "forma condensada de captar a la vez los significados que dan los sujetos de estudio a su comportamiento" y "proporciona un discurso propio al investigador" (Velasco y Díaz, 2006, p. 34). La observación, al desarrollarse en condiciones inseguras, nos obliga a modificar nuestra función y pasar a ser investigador estacionario que actúa como "mosca en la pared" (Álvarez, 2008, p. 7). Realizar una observación sigilosa, acompañado de lugareños, me permitió percibir cómo la disputa por "la plaza" da lugar a la conformación local de un Estado paralelo que sustituye al otrora Estado monopólico del uso de la violencia. Conocer las múltiples caras del Estado me permitió mostrar la incapacidad que este tiene para impartir justicia, así como su condición omisa para enfrentar los mercados y economías ilegales. ${ }^{21}$ Además, se reveló su incapacidad para hacer frente a la red de relaciones ilegales que forman los basamentos de un nuevo orden social ilegal.

La explicación de este orden violento evidenció que la vida regional es hoy más que nunca un espacio de disputa económica y política. Los grupos ilegales participan en las actividades agroproductivas y, en la última década, en la disputa electoral. Se ha empezado a transitar de una democracia de baja intensidad (De Sousa, 2006) a una democracia violenta (Ramírez, 2020). Esta conexión de la violencia articulada a un continuum de construcción regional belicosa está presente en las tres regiones antes mencionadas, donde se configuran regímenes ilegales. Por un lado, la Ciénega de Chapala se sitúa como el laboratorio de los procesos de intervención federal mediante la desecación de más de 50000 hectáreas de tierra lacustre del lago de Chapala, con un alto nivel de conflictividad social. A esta se adhieren los episodios de la Guerra Cristera en los años veinte y las disputas político-electorales en los años noventa, cuando la hegemonía priista se impuso mediante distintas acciones violentas contra los opositores. Por otro lado, la Tierra Caliente cuenta con una tradición agropecuaria destacada y es una de las regiones más productivas del

\footnotetext{
${ }^{20}$ En las tres regiones, el fenómeno de la violencia se encuentra articulado a una red de connivencia entre el crimen organizado, un amplio sector social que se beneficia del mercado ilegal y las autoridades policiales del estado, quienes participan mediante una "debilidad calculada" (Escalante, 2009). Es por ello que transitar por estas zonas difusas, bajo la sensación de que estamos vigilados, determina que nuestra estancia sea esporádica, breve y bajo la mayor discreción posible (un recuento de estas experiencias se encuentra en Ramírez, 2019a, 2019b y 2020).

${ }^{21}$ Nos referimos al narcomenudeo de droga vegetal y sintética, al control de plazas, barrios y calles, a la extorsión y el secuestro, al contrabando y a la industria de la falsificación, a la trata de personas y a toda actividad subterránea o informal sin control del Estado. La expansión de esta economía en las regiones tiende a favorecer también a múltiples sectores que desarrollan actividades legales, incluso porque se recluta personal profesionista calificado (Bataillon, 2015).
} 
estado. Además, es la zona con mayor tradición del cultivo y comercialización de droga vegetal, la cual se instituyó en una actividad importante para la subsistencia de la vida rural (Maldonado, 2010). Por último, la Chontalpa es una región sometida por los gobiernos federal y estatal mediante la imposición del Plan Chontalpa, que desmanteló física y culturalmente la forma de vida de esta región (Tudela, 1989). En los tres casos la revisión geohistórica de la convulsiva vida regional se constituye en un elemento de análisis sustancial para explicar la nueva construcción del poder local, cruzada por la violencia.

\section{Caminar sobre campos minados}

Durante largo tiempo, quienes se han acercado al estudio de la antropología y algunas otras disciplinas de las ciencias sociales le asignaron a la etnografía un valor significativo como herramienta para realizar un análisis teórico orientado a la construcción cultural de una población. De manera particular se valoró el trabajo de campo, porque permitía al investigador o investigadora "participar en la vida cotidiana de las personas que está investigando", lo que representaba "lo más semejante a la rutina de vivir" (Hammersley y Atkinson, 1994 en Ferrándiz, 2008, p. 91). Además, permitía desarrollar capacidades como la "paciencia y dedicación, atención esmerada y ferviente, observación y reflexión crítica de lo observado" (Sanmartín, 2000, p. 39).

Sin embargo, el desarrollo de la práctica etnográfica en escenarios permeados por la violencia pone a prueba este método (Castro, 2017). El solo hecho de internarse en una población que nos es ajena implica riesgos. Aunado a ello, la transversalidad de la violencia en algunas regiones de México nos obliga a extremar precauciones para reducir los riesgos que generan las dinámicas de estos contextos. En tales condiciones, la estrategia de campo debe repensarse en función de las condiciones que permean la disputa territorial, y a la vez debe revisarse permanentemente la funcionalidad de la estrategia de investigación que ponemos en marcha. Esto implica que el proceso de aprendizaje que representa trabajar bajo las lógicas de la violencia nos enfrentará también a un cúmulo de experiencias poco afortunadas que pueden involucrar y afectar a nuestros informantes, con quienes contraemos compromisos al momento de contactarlos y convertirlos en fuentes de información (Hjorth, 2018), a los cuales debemos reconocer como "sujetos con sus propias experiencias de vida, sus historias personales y las percepciones que tienen del mundo" (Rosemberg, 2019, p. 169).

Para reducir los riesgos al realizar trabajo investigativo en estas poblaciones deberá contemplarse un enfoque de seguridad. De acuerdo con Hjorth (2018), este enfoque requiere de una planeación estratégica que posibilite entender nuestra presencia espacial, ya sea como "visitante esporádico", como "nativo marginal" (Álvarez, 2008) —que regularmente propicia desconfianza- o como habitante o residente que comparte la vida de la población. La última modalidad puede influir positivamente en que quien investiga sea aceptado, aunque también lo sobreexpone a las subordinaciones violentas existentes. Sea como visitante o como habitante, el rapport o negociación para acceder a la población tiene un lugar medular en la estrategia de construcción de confianza y aceptación con los habitantes: "en el fondo, se trata de venderse a sí mismo como una persona digna de crédito que lleva a cabo un proyecto de valor" (Woods, 1987, p. 37). Esto implica mucho más que "pedir un permiso", pues penetramos en culturas grupales, invadimos espacios y vidas privadas (Stake, 2005, p. 58). El establecimiento y éxito del rapport dependerá, en primer lugar, de la manera en que logremos transparentar los objetivos con la población. En segundo lugar, dependerá de nuestra capacidad para identificar y entender los intereses, problemas 
y preocupaciones de la gente y actuar en correspondencia con ella. Esta empatía abonará a la construcción de relaciones de respeto y a mejorar el acceso a información. Además, en determinado escenario de inseguridad, la población puede fungir como fuente de advertencia, protección y apoyo para sortear la situación (Hjorth, 2018).

Dadas las condiciones de inseguridad, es necesario transitar más allá de los lugares comunes de la etnografía (pensada regularmente para escenarios menos álgidos) y plantearse la pertinencia de emplear técnicas tradicionales para obtener información. En este sentido, se vuelve esencial contemplar el diseño de una estrategia logística flexible, con cierto sigilo, supeditada a cambios repentinos y que, en casos extremos, considere la posibilidad de abandonar el trabajo como medida de seguridad para salvaguardar la integridad. Por ello, resulta indispensable pensar en el empleo de "etnografías fluidas" 22 que atiendan estos problemas "movedizos", así como restringir nuestra incursión permanente a estos espacios y optar por realizar visitas por periodos cortos o de ida y vuelta, y con cierta "frialdad empática" (Ferrándiz, 2008, p. 99).

Este empleo del enfoque etnográfico en territorios inseguros conduce a afinar estrategias de convivencia que mengüen los riesgos. Principalmente, se debe poner mayor atención en identificar los ritmos, rutas y horarios de movilidad de la gente, y en reconocer el uso del espacio para evadir zonas difusas. Además, resulta determinante estudiar y entender los códigos establecidos, tales como las formas de mirar y comportarse, y ser prudente para intervenir o dejar de hacerlo. Esto implica moverse en función de las lógicas vivenciales de la población local, sin comentar temas que exacerban el riesgo, y asumir patrones locales de conducta para proteger a quienes participan en el trabajo de campo. ${ }^{23}$

De acuerdo con Hjorth, al realizar trabajo de campo en estos territorios debe contemplarse que el riesgo o "la probabilidad de que ocurra algo dañino, y el alcance de ese daño" está presente, así como la amenaza de que cualquier cosa pueda causar daño "a la integridad física o moral o la propiedad de una persona" (2018, p. 77). Es por ello que se debe realizar un análisis del contexto espacial mediante un mapeo que identifique los factores de inseguridad, plantear un metaanálisis de los riesgos del trabajo de terreno, así como evaluar o examinar las amenazas y las formas de enfrentar este desafío.

Por su parte, Van Brabant considera necesario identificar los elementos que resultan relevantes para conocer el espacio y el entorno donde se desarrollan las actividades de la delincuencia. Esto es posible a través de un análisis situacional que se dirija a estudiar a los actores o grupos centrales con cierta capacidad de decisión que puedan afectar el quehacer investigativo en el espacio o región (Brabant et al., 2010). Este ejercicio implica, por un lado, identificar los límites y las posibles medidas de atenuación del riesgo y, por otro lado, ubicar los umbrales y las fronteras del trabajo de campo para examinar las amenazas que circundan el lugar, las consideraciones de su impacto y las posibilidades de que ocurran y nos afecten. Esto ha de realizarse mediante la identificación y ubicación de los diferentes actores y grupos locales y

\footnotetext{
${ }^{22}$ Las etnográficas fluidas se refieren a "ampliar el repertorio, ser capaces de diversificar los discursos en los cuales transmitimos el conocimiento producido para distintos tipos de fines y audiencias al tiempo que, como sugerimos al principio, modulamos las estrategias de investigación para aprehender adecuadamente problemas de evolución rápida" (Ferrándiz, 2008, p. 107).

${ }^{23}$ De acuerdo con Rosemberg, las precauciones en el trabajo de campo van desde "utilizar espacios neutrales para las entrevistas", ya que citar informantes locales puede significar una sentencia de muerte para ellos, proporcionar datos en un lugar 'localizable' puede ser peligrosos", por lo que nuestra responsabilidad académica y ética descansa en la protección de las fuentes y no en su revelación (2019, p. 168).
} 
externos claves con autoridad sobre la localidad, así como mediante la exploración de sus genealogías, sus intereses y el entretejido económico y político que los articula o los confronta. El mapeo permitirá también identificar el punto de inflexión, es decir, cuándo las condiciones cambiantes por la disputa del territorio propician una cadena de sucesos previos que generan condiciones de inseguridad aguda. Es por ello que es indispensable planificar medidas de mitigación de riesgo para cuando las previsiones resultan insuficientes, son desbordadas o se configura un peligro inminente o punto crítico ante el cual nuestra retirada del lugar es obligada (Hjorth, 2018). ${ }^{24}$

Estas rutas precautorias permitirán entender la geografía del poder y las fuerzas que se lo disputan. También, ponen a la vista los procesos de resiliencia social, la manera en que las poblaciones se ajustan a condiciones de inseguridad y cómo sus habitantes sobreviven a las secuelas y costos de la violencia. De ahí la necesidad de generar procesos investigativos estratégicos que tengan como base la responsabilidad social y una "ética localizada", (Kovats-Bernat, 2002, en Hjorth, 2018, p. 77). Se trata de una ética que atienda las recomendaciones de los lugareños sobre temas álgidos y que evite cuestionamientos asociados directamente con los actores ilegales y que motivan inseguridad. También se refiere a la adopción de patrones locales de conducta.

Se requiere, entonces, buscar otras vías de exploración como la economía política del narcotráfico. Esta rama de la economía permite identificar las transformaciones locales conectadas a procesos económicos transnacionales y la nueva correlación de fuerza regional en territorios donde el Estado no tiene el control. Además, en ciertas circunstancias permite conocer los vasos comunicantes que establecen los diversos actores legales e ilegales que forman parte de la construcción regional del Estado $-\mathrm{y}$ del proceso transicional que vivimos-. De este modo, se debe comprender que el estudio de la violencia está estrechamente vinculado a la construcción del Estado, el cual se construye en escenarios donde el poder tiene distintas manifestaciones locales $-\mathrm{y}$ nuestra presencia está inserta en ese reducido y visible espacio de disputa-. Es por ello que el análisis deberá encaminarse a exponer las conexiones de procesos en los que se insertan esos grupos ilegales. El análisis también deberá identificar los procesos de subalternidad y resiliencia social, en los que las víctimas de la violencia se constituyen en sujetos etnográficos de nuestro trabajo.

\section{Apuntes finales}

Los reajustes sociales a los que nos enfrentamos - desencadenados por la expansión de la violencia y dotados de alto riesgo en los escenarios regionales - ponen a prueba los límites de nuestro quehacer investigativo, especialmente el que está dirigido a comprender y generar nuevas narrativas sobre las diferentes conexiones y causalidades de los fenómenos violentos. La necesidad de explicar estos "escenarios tribales" ha propiciado que se multipliquen los objetos de estudio de las disciplinas de ciencias sociales. Al mismo tiempo, la transversalidad de la violencia ha hecho necesario el establecimiento de subdisciplinas, como la "antropología de la violencia" y la "sociología de la violencia". También, ha activado articulaciones

\footnotetext{
${ }^{24}$ Tuve experiencias de campo de este tipo con otros colegas en Tepalcatepec, donde, debido a la presencia de sujetos armados y al tenso ambiente que se percibía, decidimos retirarnos. Una situación similar nos tocó vivir en Felipe Carrillo Puerto (La Ruana), donde la captura de una fotografía propició malestar en el círculo de seguridad de uno de los líderes de autodefensa, acusado de connivencia con uno de los cárteles regionales de Michoacán.
} 
disciplinares que abonan al debate para definir rutas teóricas y metodológicas que expliquen de manera integral las expresiones de las violencias. Esta tarea debe incluir la construcción de medidas precautorias que permitan ubicar, distinguir, contextualizar y comparar diferentes contextos y expresiones de violencia, y que reduzcan los riesgos en estos "campos minados".

En consecuencia, los fenómenos permeados por la violencia requieren de una interpretación a través de la función y relación de los actores sociales circunscritos a estos contextos, donde la presencia del investigador cumple diversas funciones, entre ellas, ser el principal instrumento de investigación que busca mimetizarse en la población para vivir la vida cotidiana como un habitante más - práctica que empieza a ser revalorada frente a la necesidad de establecer una estrategia de seguridad personal-. En este sentido, la vivencia investigativa nos advierte en sí misma de la presencia de un conjunto de relaciones que obligan a extremar precauciones. Se ha vuelto imprescindible afinar la precisión en las estrategias para anticipar y enfrentar situaciones de incertidumbre y dificultades emergentes. Es necesario también construir una narrativa previsora que resguarde nuestra integridad y la de los sujetos involucrados en la investigación y que retome las experiencias de la violencia, así como las narrativas de los sujetos de la violencia. En tal sentido, como bien menciona Jimeno (2007, p. 188), es necesario "ahondar en el terreno de la subjetividad mediante los relatos sobre experiencias de violencia, pues quizá arrojen luz sobre el campo político", y porque nos acerca a "la posibilidad de identificarnos con las víctimas, permite comprender su membresía a la comunidad y restablecer lazos para la acción ciudadana”.

Por todo lo anterior, resulta sustancial para la persona que investiga desentrañar las nuevas formas de poder ilegal, las jerarquías y luchas de poder que están en juego en las experiencias de violencia de este tipo. Para ello han de construirse narrativas que no solo visibilicen las injusticias que viven quienes están involucrados en la investigación, sino que también permitan entender la subjetividad de cada persona en escenarios de violencia y diagramar posibles salidas que contribuyan a la construcción de una "cultura de la paz". Hay que mencionar, además, que esto debe hacerse mediante una nueva lectura y uso crítico de la teoría, en tanto instrumento de reflexión de la realidad, que acompañe el análisis de los insumos obtenidos en nuestra labor investigativa.

\section{Referencias}

Álvarez Álvarez, C. (2008). La etnografía como modelo de investigación en educación. Gazeta de Antropología, (24), 1-15. http://www.gazeta-antropologia.es/?p=2347

Arteaga Botello, N., y Arzuaga Magnoni, J. (2017). Sociologías de la violencia. Estructuras, sujetos, interacciones y acción simbólica. Facultad Latinoamericana de Ciencias Sociales.

Astorga, L. (2009). Transición democrática, organizaciones de traficantes y lucha por la hegemonía. En R. Benítez, A. Rodríguez y A. Rodríguez (coords.), Atlas de la seguridad y la defensa de México 2009 (pp. 105-109). Colectivo de Análisis de la Seguridad con Democracia.

Auyero, J. (2007). La zona gris. Violencia colectiva y política partidaria en la Argentina contemporánea. Siglo XXI Editores.

Azula Díaz del Castillo, S. del P. (2017). ¿Cuándo gobiernan los criminales? un análisis comparado del Clan del Golfo y el Cártel de los Zetas [monografía para optar al título de Internacionalista, Universidad de] Rosario].

Bataillon, G. (2015). Narcotráfico y corrupción: las formas de la violencia en México en el siglo XXI. Nueva Sociedad, (255), 54-68. https://nuso.org/articulo/narcotrafico-y-corrupcion-las-formas-de-la-violencia-enmexico-en-el-siglo-xxi/

Brabant, K. Van et al. (2010). Operational Security Management in Violent Environments. Humanitarian Practice Network; Overseas Development Institute. 
Bonilla, D. (2007). Presentación. En R. I. Rotbert, C. Clapham y J. Herbst (coords.), Los Estados fallidos o fracasados: un debate inconcluso y sospechoso (pp. 13-20). Siglo del Hombre Editores; Universidad de los Andes; Pontificia Universidad Javeriana.

Bourgois, P. (2010). En busca de respeto. Vendiendo crack en Harlem. Siglo XXI Editores.

Briscoe, I. (2008). La proliferación del "estado paralelo". FRIDE, (71), 1-18.

Castellanos, L. (2015, 19 de abril). Fueron los federales. Aristegui Noticias. https://aristeguinoticias.com/1904/ investigaciones-especiales/fueron-los-federales/

Castro Neira, Y. (2017). Etnografías de la violencia. Dilemas para hacer y pensar las etnografías en zonas de guerra, violencia y conflicto. En Y. Castro Neira y A. Blazquez (coords.), Micropolíticas de la violencia. Reflexiones sobre el trabajo de campo en contextos de guerra, conflicto y violencia (pp. 60-70). Laboratorio Mixto Internacional. Movilidades; Gobernanza y Recursos en la Cuenca Mesoamericana. Cuadernos de Trabajo de MESO, 5. http://ri.ibero.mx/bitstream/handle/ibero/1293/CNYA_Doct_0l.pdf?sequence=1\&eisAllowed=y

Cavarero, A. (2009). Horrorismo. Nombrando la violencia contemporánea. Universidad Autónoma MetropolitanaUnidad Iztapalapa; Anthropos.

Cisneros, J. L. (2015). Visiones contemporáneas de la violencia. Universidad Autónoma Metropolitana; Eón.

Das, V. (1997). Language and Body: Transactions in the Construction of Pain. En A. Kleinma, V. Das y M. M. Lock (eds.), Social Suffering (pp. 67-91). University of California Press.

De Sousa Santos, B. (2006). Renovar la teoría crítica y reinventar la emancipación social. Consejo Latinoamericano de Ciencias Sociales.

En la última década, los homicidios dolosos en Michoacán se dispararon un 161\% (2020, 23 de septiembre). La Voz de Michoacán. https://www.lavozdemichoacan.com.mx/michoacan/en-la-ultima-decada-loshomicidios-en-michoacan-se-dispararon-en-161-5/

Escalante Gonzalbo, F. (2009). Violencia, narcotráfico y Estado. Nueva Sociedad, (220), 84-96. https://nuso.org/ articulo/violencia-narcotrafico-y-estado/

Estrada, G., Hinojosa, D., y Badillo, D. (2019, 18 de agosto). Violencia, sin tregua por años en Michoacán. El Economista, 5. https://www.eleconomista.com.mx/politica/Violencia-sin-tregua-por-anos-enMichoacan-20190818-0082.html

Ferrándiz, F., y Feixa Pampols, C. (2004). Una mirada antropológica sobre las violencias. Alteridades, (27), 159174. https://alteridades.izt.uam.mx/index.php/Alte/article/view/317

Ferrándiz, F. (2008). La etnografía como campo de minas: De las violencias cotidianas a los paisajes posbélicos. En M. Bullen y C. Díez (coords.), Retos teóricos y nuevas prácticas (pp. 89-115). Ankulegi.

Galtung, J. (2004). Violencia, guerra y su impacto. Sobre los efectos visibles e invisibles de la violencia. Polylog. Foro para filosofía intercultural, 5. http://them.polylog.org/5/fgj-es.htm

Giménez, G. (2017). Introducción. En G. Giménez y R. Jiménez (coords.), La violencia en México a la luz de las ciencias sociales (pp. 9-34). Instituto de Investigaciones Sociales-Universidad Nacional Autónoma de México. http://ru.iis.sociales.unam.mx/bitstream/IIS/5289/1/violencia_mexico_completo.pdf

Gledhill, J. (2008). El poder y sus disfraces. Bellaterra.

González González, F. E. (2014). Poder y violencia en Colombia. Odecofi; Cinep.

Gutiérrez, A. (2020, 24 de agosto). Violencia en Michoacán desplazó a 704 personas en 2019. El Sol de Morelia. https://www.elsoldemorelia.com.mx/local/violencia-en-michoacan-desplazo-a-704-personasen-2019-5667708.html

Hjorth Boisen, S. V. (2018). Evaluación y reducción de riesgo en el trabajo de campo. Alteridades, (28), 73-84. https://alteridades.izt.uam.mx/index.php/Alte/article/view/983

Jackman, M. (2002). Violence in Social Life. Annual Review of Sociology, (28), 387-415.

Jiménez-Ocampo, S. (2008). Etnografía y crisis: algunos debates y una práctica de investigación en contextos de violencia. Nómadas, (29), 34-49. https://bit.ly/2V2cDRO

Jimeno, M. (2007). Lenguaje, subjetividad y experiencias de violencia. Antípoda. Revista de Antropología y Arqueología, (5), 169-190. https://doi.org/10.7440/antipoda5.2007.08

Le Cour, R. (2019). "Pueblo chico, infierno grande". Territorialidad e intermediación política: las autodefensas de Michoacán". En Violencia inseguridad y Estado de derecho (pp. 153-178). El Colegio de Michoacán.

Maldonado Aranda, S. (2010). Los márgenes del Estado mexicano. Territorios ilegales, desarrollo y violencia en Michoacán. El Colegio de Michoacán.

Maldonado Aranda, S. (2013). Desafíos etnográficos en el estudio de la violencia. Experiencias de una investigación. Avá. Revista de Antropología, (22), 123-144. https://www.redalyc.org/pdf/1690/169036843006.pdf 
Maldonado Aranda, S. (2014). Despejando caminos inseguros. Itinerarios de una investigación sobre la violencia en México. Alteridades, (47), 63-76. https://alteridades.izt.uam.mx/index.php/Alte/article/ view/779

Martínez Pacheco, A. (2016). La violencia. Conceptualización y elementos para su estudio. Política y Cultura, (46), 7-31. https://polcul.xoc.uam.mx/index.php/polcul/article/view/1300/1275

Nordstrom, C. (1997). A different kind of war story. University of Pennsylvania Press.

Pansters Wil G. (2019). iTuvimos que pagar para poder vivir! Soberanías encontradas en el México Violento. En Violencia inseguridad y Estado de derecho (pp. 94-121). El Colegio de Michoacán.

Pearce, J. (2019). Introducción. Un aporte conceptual y empírico para resignificar la seguridad en México. En G. Kloppe-Santamaría y A. Abello Colak (eds.), Seguridad humana y violencia crónica en México. Nuevas lecturas y propuestas desde abajo (pp. 5-34). Instituto Tecnológico Autónomo de México; Centro de Investigación y Docencia Económicas; MAPorrúa.

Pontón, D. (2013). La economía del narcotráfico y su dinámica en América Latina. Íconos. Revista de Ciencias Sociales, (47), 135-153. https://doi.org/10.17141/iconos.47.2013.853

Ramírez Sánchez, R. D. (2019a). Del edén al infierno: inseguridad y construcción estatal en Tabasco. LiminaR. $\begin{array}{lllll}\text { Estudios Sociales } & y & \text { Humanísticos, } & \text { 17(2), }\end{array}$ HTTPS: DOI.ORG/10.29043/LIMINAR.V17I2.690

Ramírez Sánchez, R. D. (2019b). Conflictos regionales y Estado en el Occidente michoacano. En D. Chávez Jiménez y T. Aguilar (coords.), Comunidad y territorio: el caso del Occidente michoacano (pp. 41-62). Universidad Nacional Autónoma de México.

Ramírez Sánchez, R. D. (2020). Elecciones Michoacán, 2018: entre el conflicto y la violencia. Apuntes Electorales, (63), 71-110. https://aelectorales.ieem.org.mx/index.php/ae/article/view/735

Reguillo, R. (2012). De las violencias: caligrafía y gramática del horror. Desacatos, (40), 33-46. https://doi. org $/ 10.29340 / 40.254$

Rodríguez Guillén, R. (2012). Crisis de autoridad y violencia social; los linchamientos en México. Polis, 8(2), 43-74. https://revistas-colaboracion.juridicas.unam.mx/index.php/polis/article/view/17032

Rosemberg, F. (2019). La etnografía en tiempos de violencia. Cuicuilco Revista de Ciencias Antropológicas, (76), 153 174. http://www.scielo.org.mx/pdf/crca/v26n76/0185-1659-cuicui-26-76-153.pdf

Rotberg, R. I., Clapham C., y Herbst J. (coords.) (2007). Los Estados fallidos o fracasados: un debate inconcluso y sospechoso. Siglo del Hombre Editores; Universidad de los Andes; Pontificia Universidad Javeriana.

Sanmartín Arce, R. (2000). Etnografía de los valores. Teoría de la Educación, (12), 129-141.

Schinkel, W. (2013). Regimes of Violence and the Trias Violentiae. European Journal of Social Theory, 16(3), 310-325.

Secretariado Ejecutivo del Sistema Nacional de Seguridad Pública (SESNSP) (2020). Información de violencia contra las mujeres.

Segato, R. (2013). La escritura en el cuerpo de las mujeres asesinadas en Ciudad Juárez. Tinta Limón.

Stake, R. (2005). Investigación con estudio de casos. Morata.

Tedesco, L. (2007). El Estado en América Latina. iFallido o en proceso de formación? Fride.

Trejo, G., y Ley, S. (2015, 1 de febrero). Municipios bajo fuego (1995-2015). Nexos, Sociedad, Ciencia y Literatura. https://www.nexos.com.mx/?p=24024

Tudela, F. (coord.) (1989). La modernización forzada del trópico: el caso de Tabasco. El Colegio de México; Instituto Politécnico Nacional; Centro de Investigación y de Estudios Avanzados; Federación Internacional de Institutos de Estudios Avanzados; Instituto de Investigaciones de las Naciones Unidas para el Desarrollo Social.

Velasco, H., y Díaz de Rada, Á. (2006). La lógica de la investigación etnográfica. Un modelo de trabajo para etnógrafos de escuela. Trotta.

Woods, P. (1987). La escuela por dentro. La etnografía en la investigación educativa. Paidós; Ministerio de Educación y Ciencia.

Zavaleta Betancourt, J. A. (2017). El campo de los estudios de la violencia en México. Anuario Latinoamericano Ciencias Politicas y Relaciones Internacionales, (4), 41-60. https://journals.umcs.pl/al/article/view/5413/0

Žižek, S. (2009). Sobre la violencia. Seis reflexiones marginales. Paidós. 


\section{Referencias}

Arribas, A. (2018). Intranet para la gestión del conocimiento y la comunicación interna. Razón y Palabra, 22(1100), 406-413. https://www.revistarazonypalabra.com/index.php/ryp/article/view/1163

Capriotti, P. (1999). Planificación estratégica de la imagen corporativa. Ariel.

Dörnyei, Z. (2007). Research methods in applied linguistics: Quantitative, qualitative, and mixed methodologies. Oxford University Press.

Fernández, M., y Savall, H. (2004). El modelo de gestión socio-económica en organizaciones mexicanas. Universidad Autónoma Metropolitana-Iztapalapa.

Fuentes, O. (2015). La organización escolar. Fundamentos e importancia para la dirección en la educación. VARONA, Revista Cientifico-Metodológica, (61), 1-12. https://www.redalyc.org/pdf/3606/360643422005.pdf

Fundación SERES (2014, 6 de noviembre). 10 consejos para comunicar con éxito la RSE. Compromiso RSE. https:/www.compromisorse.com/rse/2014/11/06/10-consejos-para-comunicar-con-exito-la-rse-/

Garbanzo-Vargas, G. (2016). Desarrollo organizacional y los procesos de cambio en las instituciones educativas, un reto de la gestión de la educación. Revista Educación. 40(1), 67-87. http://dx.doi.org/10.15517/revedu.v40il.22534

Hernández Flores, H. G. (2018). De la comunicación institucional a la responsable, un beneficio auténtico para las organizaciones. ObservaRSE. http://www.observarse.com/2018/01/04/comunicacion-institucionalresponsable-beneficio-organizaciones/

Instituto de Socioeconomía de las Empresas y Organizaciones (ISEOR) (s.f.). El modelo de gestión socioeconómica en organizaciones mexicanas. http://164.132.163.82/SiteIseor/ouvrages_fiche.asp?code=LIV2004MFE001

Jaksa, J. (1996). Responsible Communication: Ethical Issues in Business, Industry, and the Professions. Hampton Press.

Meseguer, J., García, M., y Hernández, R. (2018). Manual de comunicación creativa y responsable. Universidad Católica San Antonio, Lógica Ecommerce. https:/logicaecommerce.com/formacion/wp-content/ uploads/2018/12/guia-de-la-comunicacion-responsable-final.pdf

Orozco-Toro, J., y Ferré-Pavia, C. (2019). Los intangibles de la marca y su efecto en la reputación corporativa. La evaluación de toda cadena de valor de Televisión de Catalunya. Revista de Comunicación, 18(1), 111-134. https://doi.org/10.26441/RC18.1-2019-A6

Oyarvide-Ramírez, H., Reyes-Sarria, E., y Montaño-Colorado, M. (2017). La comunicación interna como herramienta indispensable de la administración de empresas. Revista Científica Dominio de las Ciencias, 3(4), 296-309. http://dx.doi.org/10.23857/dc.v3i4.687

Paladines, F., Valarezo, K., y Yaguache, J. (2013). La comunicación integral, un factor determinante en la gestión de la empresa ecuatoriana. Signo y Pensamiento, 32(63), 110-128. https://doi.org/10.11144/Javeriana.syp32-63.cifd

Pertúz, F. (2018). Liderazgo transformacional en empresas sociales desde la perspectiva ética de la responsabilidad social empresarial. Telos, 20(2), 377-388. https://dialnet.unirioja.es/servlet/articulo?codigo=6436416

Ramírez, R., y Hugueth, A. (2017). Modelo de comunicación productiva para las organizaciones de salud pública en Venezuela. Opción, 33(83), 305-335. https://www.redalyc.org/pdf/310/31053772011.pdf

Remund, D. L. (2015). The ART of Responsible Communication: Leading with Values Every Day. Business Expert.

Rincón, Y., y Ramírez, R. (2018). Comunicación responsable en las organizaciones. En Quintero, M., y Sánchez, M. (eds.). Responsabilidad social corporativa. Una mirada integral en América Latina (pp. 93-100). Editorial Univalle. https://www.researchgate.net/publication/328160423_Comunicacion_responsable_en_las_organizaciones

Rincón, Y., Sukier, H., Contreras, J., y Ramírez, R. (2019). Estrategias de comunicación responsable para pequeñas y medianas empresas. Opción, 35(89-2), 1208-1234. https://repositorio.cuc.edu.co/handle/11323/5377

Rivera Salas, P., y Hernández Flores, H. (2020). Comunicación responsable en infografías digitales publicadas por empresas farmacéuticas. Correspondencias e Análisis, (12). https://doi.org/10.24265/cian.2020.nl2.02

Rodríguez-Cruz, Y., y Pinto, M. (2018). Modelo de uso de información para la toma de decisiones estratégicas en organizaciones de información. Transinformação, 30(1), 51-64. https://doi.org/10.1590/231808892018000100005

Tapia-Carreto, A., Mendoza, R., y Martínez, O. (2016). Reconociendo el desarrollo de competencias sociodiscursivas en la universidad. AgoradeHeterodixias, 2(2), 22-36. https://redib.org/Record/oai_articulo2905109reconociendo-el-desarrollo-de-competencias-socio-discursivas-en-la-universidad

Vásquez-Rizo, F.-E., y Gabalán-Coello, J. (2015). Información y ventaja competitiva. Coexistencia exitosa en las organizaciones de vanguardia. Profesional de la Información, 24(2), 149-156. https://doi.org/10.3145/epi.2015.mar.08

Villalba, J. (2015, 9 de marzo). Comunicación responsable: una oportunidad para ganar. Telefónica Empresas. https://empresas.blogthinkbig.com/comunicacion-responsable-una-oportunidad-para-ganar/ 